\title{
Kalman Filtering with Uncertain Process and Measurement Noise Covariances with Application to State Estimation in Sensor Networks
}

\author{
Ling Shi*, Karl Henrik Johansson ${ }^{\dagger}$ and Richard M. Murray*
}

\begin{abstract}
Distributed state estimation under uncertain process and measurement noise covariances is considered. An algorithm based on sensor fusion using Kalman filtering is investigated. It is shown that if the covariances are decomposed into a known nominal covariance plus an uncertainty term, then the uncertainty of the actual estimation error covariance for the Kalman filter grows linearly with the size of the uncertainty term. This result is extended to the sensor fusion scheme to give an upper bound on the actual error covariance for the fused state estimate. Examples are provided to illustrate how the theory can be applied in practice.
\end{abstract}

\section{INTRODUCTION}

Modern sensor and communication technologies enable a variety of new networked monitoring and control applications. In many of these applications, there is an economic incentive towards using off-the-shelf sensors and standardized communication solutions. A consequence of this is that the individual hardware components might be of relatively low quality and that communication resources are quite limited. These problems can sometimes be efficiently tackled through intelligent software implementations. A particular instance of this approach is the distributed estimation algorithms that take the added uncertainties and resource constraints into account discussed in this paper.

Networked sensor and control applications are found in a growing number of areas, including automobiles, autonomous vehicles, environmental monitoring, industrial automation, power distribution, space exploration, surveillance, and transportation. Let us discuss three concrete examples that have motivated our work. Alice is an autonomous vehicle that was developed at California Institute of Technology for the 2005 DARPA Grand Challenge [1]. The sensors mounted on Alice include an inertial measurement unit (IMU), global positioning system (GPS), velocity and measurement range sensors, and monocular vision. To allow the vehicle to autonomously navigate through its environment, sensor data are fused to provide Alice with an estimate of its own state and of the environment around it. The heterogeneous set of sensors is connected with the computation platform through

*: Control and Dynamical Systems, California Institute of Technology, Pasadena, CA 91106. Email: \{shiling, murray\}@cds.caltech.edu.

$\dagger$ : School of Electrical Engineering, Royal Institute of Technology, Stockholm, Sweden. Email: kallej@ee.kth.se.

Tel: (626) 395-2313, Fax: (626) 395-6170

The work by L. Shi and R. M. Murray is supported in part by AFOSR grant FA9550-04-1-0169. The work by K. H. Johansson is supported by the Swedish Research Council and the Swedish Foundation for Strategic Research through an Individual Grant for the Advancement of Research Leaders. an Ethernet local area network providing an architecture for networked estimation and control.

Another class of examples is enabled by the standardized controller area network (CAN), which is used in most cars and trucks today and has revolutionized the automotive industry, where embedded control has grown from stand-alone systems to highly integrated and networked systems [2]. Look-ahead driver assistance systems and control systems for improved fuel efficiency of auxiliary units are instances of currently developed technologies in heavy duty vehicles based on on-board sensors, online digitized maps, and various traffic information systems [3], [4]. As the number of these type of systems grow, the resource constraints of the CAN and the implementation complexity need to be considered in the design on the distributed estimation and control algorithms. The results of this paper is applied to a road grade estimation problem for a look-ahead cruise controller.

Kalman filtering [5] is a well established methodology for model-based fusion of sensor data [6]-[9]. Despite the great success of this approach, with extensions to for instance nonlinear and decentralized systems, only recently the specific characteristics of the sensing and communication in networked estimation systems have been considered. Estimation under certain communication constraints has been considered, e.g., bandwidth limitation [10] and packet loss [11], [12]. The shared communication resource can be efficiently controlled through an active scheduling mechanism [13].

The traditional Kalman filter requires exact knowledge of the plant model and the statistics of the process noise and measurement noise. In sensor network applications this information is often uncertain due to hardware variability, sensor drift, unmodeled plant dynamics etc. Kalman filtering with unknown process noise and measurement noise covariances is a classical problem, e.g., [14]. A variety of methods have been developed to simultaneously estimate the covariances with the state [8]. An alternative approach is to consider the influence on the estimation from unknown but fixed errors in the noise statistics. This is the approach taken in this paper, where bounds are given on the estimation error both for the conventional Kalman filter but also for a sensor fusion scheme suitable for a networked implementation.

The main contribution of this paper is on deriving a new performance bound for a sensor fusion scheme that explicitly takes the model uncertainty of the underlying processes and sensors into account. Based on the classical Kalman filter, the estimation error covariance is computed for given uncertainties of the process and measurement noise covariances. 


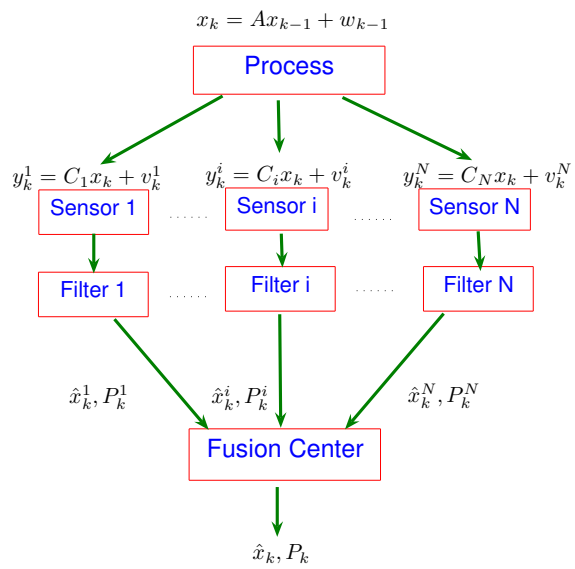

Fig. 1. Sensor fusion block diagram

It is shown that if the covariances are decomposed into a known nominal covariance plus an uncertainty term, then the uncertainty of the actual estimation error covariance for the Kalman filter grows linearly with the size of the uncertainty term. It is also shown how these results can be extended to a networked system, where partial estimates of the plant state are fused in a central node. An upper bound on the actual error covariance for the fused state estimate is derived. Examples are provided to illustrate how the theory can be applied in practice.

The outline of the paper is as follows. The state estimation problem is formulated in Section II followed by some preliminaries on Kalman filtering in Section III. A theorem on the estimation error covariance for uncertain process and measurement noise covariances is given in Section IV. The result is utilized in Section $\mathrm{V}$, where the main theorem of the paper is presented. It states the influence of the uncertain noise covariances on a commonly used sensor fusion scheme from the literature. Section VI illustrates the theory through some simulated examples of increasing complexity. The paper is concluded in Section VII.

\section{Problem Set UP}

Consider a sensor network consisting of $N \geq 1$ sensors that take measurements for the same process and send their individual estimate of the state to a remote fusion center (see Figure 1). The process dynamics is given by

$$
x_{k}=A x_{k-1}+w_{k-1}
$$

and the measurement equation of each sensor $i$ is given by

$$
y_{k}^{i}=C_{i} x_{k}+v_{k}^{i}
$$

where $i=1, \cdots, N, k=1,2, \cdots$ and $w_{k-1}, v_{k}^{i}$ are white gaussian noises with covariance matrices $Q \geq 0$ and $R_{i}>0$ respectively. Assume

$$
Q=Q_{0}+\lambda \Psi \Psi^{\prime}, R_{i}=R_{0 i}+\gamma_{i} \Phi_{i} \Phi_{i}^{\prime},
$$

where $Q_{0} \geq 0, R_{0 i}>0$ are known, but $\lambda \Psi \Psi^{\prime}$ and $\gamma_{i} \Phi_{i} \Phi_{i}^{\prime}$ are unknown. Each sensor $i$ runs a Kalman filter for generating its state estimate $\hat{x}_{k}^{i}$ at time $k$ with error covariance $P_{k}^{i}$. Those estimates and error covariances are then sent to a fusion center to obtain a final state estimate.

For each sensor $i$, if there is no uncertainty in the noise covariance, i.e., $\lambda \Psi \Psi^{\prime}=0$ and $\gamma_{i} \Phi_{i} \Phi_{i}^{\prime}=0$, then the Kalman filter provides an optimal estimate of the state $x_{k}$. It gives an efficient means to estimate the state of the process, in a way that minimizes the mean of the squared error.

On the other hand, if the exact noise covariance is not known, i.e., $\lambda \Psi \Psi^{\prime} \neq 0$ or $\gamma_{i} \Phi_{i} \Phi_{i}^{\prime} \neq 0$, it is clear that the output from the Kalman filter based on $A, Q_{0}, C, R_{0 i}$, which we denote as the computed error covariance does not give the true error covariance.

We are interested in how the uncertainties in the process and measurement noise covariances from each individual sensor affect the fused state estimate. The estimate is unbiased but the computed error covariance will not converage to the true value. We provide bounds on the steady state value of the covariance.

We first study the one-sensor case and find the relationship between the actual error covariance and the uncertainties associated with the process and measurement noise covariances. For this part, we drop the subscript $i$ in Equ (2) and write the measurement noise as $v_{k}$ which has covariance $R=R_{0}+\gamma \Phi \Phi^{\prime}$. We then apply the result to the multiple sensor fusion problem with details in Section V.

\section{PRELIMINARIES}

We briefly introduce the essence of Kalman filter in this section. Before we give the recursive equations for Kalman filter, let us first define the following quantities

$$
\begin{aligned}
\hat{x}_{k}^{-} & \triangleq \mathrm{E}\left[x_{k} \mid \mathcal{Y}_{k-1}\right] \\
e_{k}^{-} & \triangleq x_{k}-\hat{x}_{k}^{-} \\
P_{k}^{-} & \triangleq \mathrm{E}\left[e_{k}^{-} e_{k}^{-{ }^{\prime}} \mid \mathcal{Y}_{k-1}\right] \\
\hat{x}_{k} & \triangleq \mathrm{E}\left[x_{k} \mid \mathcal{Y}_{k}\right] \\
e_{k} & \triangleq x_{k}-\hat{x}_{k} \\
P_{k} & \triangleq \mathrm{E}\left[e_{k} e_{k}^{\prime} \mid \mathcal{Y}_{k}\right]
\end{aligned}
$$

where $\mathcal{Y}_{k} \triangleq\left[y_{1}, \cdots, y_{k}\right]$.

When $\lambda=0$ and $\gamma=0$, the Kalman filter computes the state estimate and the error covariance as follows

$$
\begin{aligned}
\hat{x}_{k}^{-} & =A \hat{x}_{k-1}, \\
P_{k}^{-} & =A P_{k-1} A^{\prime}+Q_{0} . \\
\hat{x}_{k} & =A \hat{x}_{k-1}+K_{k}\left(y_{k}-C A \hat{x}_{k-1}\right), \\
K_{k} & =P_{k}^{-} C^{\prime}\left[C P_{k}^{-} C^{\prime}+R_{0}\right]^{-1} \\
P_{k} & =\left(I-K_{k} C\right) P_{k}^{-} .
\end{aligned}
$$

It can be shown that $P_{k}^{-}$evolves as

$$
\begin{aligned}
P_{k}^{-}= & A P_{k-1}^{-} A^{\prime}+Q_{0} \\
& \quad-A P_{k-1}^{-} C^{\prime}\left[C P_{k-1}^{-} C^{\prime}+R_{0}\right]^{-1} C P_{k-1}^{-} A^{\prime},
\end{aligned}
$$


and $P_{k}$ evolves as

$$
\begin{array}{r}
P_{k}=\left(K_{k} C A-A\right) P_{k-1}\left(K_{k} C A-A\right)^{\prime}+K_{k} R_{0} K_{k}^{\prime} \\
+\left(K_{k} C-I\right) Q_{0}\left(K_{k} C-I\right)^{\prime} .
\end{array}
$$

\section{Kalman Filter with Uncertainties in Process} and Measurement Noise Covariance

In this section, we consider the process and measurement noise covariances take the form as stated in Section II

$$
Q=Q_{0}+\lambda \Psi \Psi^{\prime}, \quad R=R_{0}+\gamma \Phi \Phi^{\prime} .
$$

Only $Q_{0}$ and $R_{0}$ are used in the Kalman filter update equations as $\lambda \Psi \Psi^{\prime}$ and $\gamma \Phi \Phi^{\prime}$ are unknown. As a result the computed $P_{k}$ from (9)-(13) is not the actual error covariance. We define the latter as $\bar{P}_{k}$. Notice that the state estimate $\hat{x}_{k}$ still converges to the mean of the process state $x_{k}$ as the estimate is unbiased. Define

$$
P_{\infty} \triangleq \lim _{k \rightarrow \infty} P_{k}^{-}, K \triangleq \lim _{k \rightarrow \infty} K_{k}
$$

which satisfy

$$
\begin{aligned}
P_{\infty}= & A P_{\infty} A^{\prime}+Q_{0} \\
& -A P_{\infty} C^{\prime}\left[C P_{\infty} C^{\prime}+R_{0}\right]^{-1} C P_{\infty} A^{\prime}, \\
K= & P_{\infty} C^{\prime}\left[C P_{\infty} C^{\prime}+R_{0}\right]^{-1} .
\end{aligned}
$$

Further define, if the limit exists,

$$
\bar{P}_{\infty}(\lambda, \gamma)=\bar{P}_{\infty} \triangleq \lim _{k \rightarrow \infty} \bar{P}_{k}
$$

Then we have the following result.

Theorem 1: Assume $(C, A)$ observable and $\left(A, Q_{0}^{\frac{1}{2}}\right)$ controllable. Then $\bar{P}_{\infty} \geq 0$ is the unique solution to the following discrete time Lyapunov equation

$$
\begin{aligned}
\bar{P}_{\infty}= & (K C A-A) \bar{P}_{\infty}(K C A-A)^{\prime}+K R_{0} K^{\prime} \\
& +\lambda(K C \Psi-\Psi)(K C \Psi-\Psi)^{\prime}+\gamma K \Phi \Phi^{\prime} K^{\prime} \\
& +(K C-I) Q_{0}(K C-I)^{\prime}
\end{aligned}
$$

Moreover,

$$
\bar{P}_{\infty}(\lambda, \gamma)=\bar{P}_{\infty}(0,0)+\lambda V_{1}+\gamma V_{2}
$$

where

$$
\begin{aligned}
M & =(K C \Psi-\Psi)(K C \Psi-\Psi)^{\prime} \\
V_{1} & =\sum_{k=0}^{\infty}(K C A-A)^{k} M(K C A-A)^{\prime k} . \\
V_{2} & =\sum_{k=0}^{\infty}(K C A-A)^{k} K \Phi \Phi^{\prime} K^{\prime}(K C A-A)^{\prime k}
\end{aligned}
$$

The second part of the theorem tells us the actual error covariance grows linearly as a function of the uncertainties of the process and measurement noise covariances. We need a few tools before proving this theorem.

Lemma 2: Consider the discrete-time Lyapunov equation

$$
H X H^{\prime}-X+M=0,
$$

where $M$ is hermitian. Then the following are true.

1) If $H$ is stable, then $X$ is unique and Hermitian and

$$
X=\sum_{k=0}^{\infty} H^{k} M H^{\prime k} \text {. }
$$

2) If $H$ is stable and $M>0$ (or $M \geq 0$ ), then $X$ is unique, Hermitian and $X>0$ (or $X \geq 0$ ).

Proof: The proof is omitted as this is from the standard analysis of discrete time Lyapunov equation.

Lemma 3: Suppose that $X \in \mathbb{R}^{m \times n}$ and $Y \in \mathbb{R}^{n \times m}$ with $m \leq n$. Then $Y X$ has the same eigenvalues as $X Y$, counting multiplicity, together with an additional $n-m$ eigenvalues equal to 0 .

Proof: See [15], Theorem 1.3.20, page 53.

Lemma 4: Consider the discrete time Riccati Equation (14) where $P_{0}^{-} \geq 0$ is arbitrary. Assume the pair $(C, A)$ is observable, and $\left(A, Q_{0}^{\frac{1}{2}}\right)$ is controllable. Then

1) There exists a $P \geq 0$ such that for all $P_{0}^{-} \geq 0$,

$$
\lim _{k \rightarrow \infty} P_{k}^{-}=P \text {. }
$$

Furthermore, $P$ is the unique solution of the algebraic matrix equation

$$
P=A\left(P-P C^{\prime}\left[C P C^{\prime}+R_{0}\right]^{-1} C P\right) A^{\prime}+Q_{0}
$$

within the class of positive semidefinite symmetric matrices. 2) The eigenvalues of the matrix

$$
D=A^{\prime}-C^{\prime}\left[C P C^{\prime}+R_{0}\right]^{-1} C P A^{\prime}
$$

are strictly within the unit circle.

Proof: See [16], Proposition 4.4.1, page 145, with slight modification of the parameters (i.e., replacing $A$ with $A^{\prime}$ and $B$ with $C^{\prime}$.

We have defined $\bar{P}_{k}$ as the actual error covariance and we will derive the update equation for $\bar{P}_{k}$ below. Notice that as long as the parameters $\left(A, C, Q_{0}, R_{0}\right)$ are given, the a priori estimation error covariance $P_{k}^{-}$is updated recursively according to Equ (14), and the Kalman gain $K_{k}$ is then updated according to Equ (12). Those two update steps do not depend on $\lambda \Psi \Psi^{\prime}$ or $\gamma \Phi \Phi^{\prime}$.

From Equ (11), we expand $y_{k}-C A \hat{x}_{k}$ to get

$$
\hat{x}_{k}=A \hat{x}_{k-1}+K_{k}\left(C A e_{k-1}+C w_{k-1}+v_{k}\right),
$$

where as before $K_{k}$ is given by Equ (12) and $P_{k}^{-}$is given by Equ (14). We can then write

$$
e_{k}=A e_{k-1}+w_{k-1}-K_{k}\left(C A e_{k-1}+C w_{k-1}+v_{k}\right) .
$$

Since the actual process and measurement noise covariances are given by

$$
\mathrm{E}\left[w_{k-1} w_{k-1}^{\prime}\right]=Q_{0}+\lambda \Psi \Psi^{\prime}, \quad \mathrm{E}\left[v_{k} v_{k}^{\prime}\right]=R_{0}+\gamma \Phi \Phi^{\prime},
$$

we have

$$
\begin{aligned}
\bar{P}_{k}= & \mathrm{E}\left[e_{k} e_{k}^{\prime}\right] \\
= & A \bar{P}_{k-1} A^{\prime}+Q_{0}+\lambda \Psi \Psi^{\prime}+K_{k}\left(C A \bar{P}_{k-1} A^{\prime} C^{\prime}+\right. \\
& \left.+C Q_{0} C^{\prime}+\lambda C \Psi \Psi^{\prime} C^{\prime}+R_{0}+\gamma \Phi \Phi^{\prime}\right) K_{k}^{\prime} \\
& -A \bar{P}_{k-1} A^{\prime} C^{\prime} K_{k}^{\prime}-K_{k} C A \bar{P}_{k-1} A^{\prime} \\
& -\left(Q_{0}+\lambda \Psi \Psi^{\prime}\right) C^{\prime} K_{k}^{\prime}-K_{k} C\left(Q_{0}+\lambda \Psi \Psi^{\prime}\right)
\end{aligned}
$$


After some simplification, we can write $\bar{P}_{k}$ as

$$
\begin{aligned}
\bar{P}_{k}= & \left(K_{k} C A-A\right) \bar{P}_{k-1}\left(K_{k} C A-A\right)^{\prime}+K_{k} R_{0} K_{k}^{\prime} \\
& +\left(K_{k} C-I\right) Q_{0}\left(K_{k} C-I\right)^{\prime}+\gamma K_{k} \Phi \Phi^{\prime} K_{k}^{\prime} \\
& +\lambda\left(K_{k} C \Psi-\Psi\right)\left(K_{k} C \Psi-\Psi\right)^{\prime} .
\end{aligned}
$$

Now we are ready to prove Theorem 1 .

Proof: First notice that if $(C, A)$ observable and $\left(A, Q_{0}^{\frac{1}{2}}\right)$ controllable, Equ (16)-(17) have unique solutions $P_{\infty}$ and $K$ from part one of Lemma 4 . It remains to show that $\bar{P}_{\infty}$ exists for any $\lambda \Psi \Psi^{\prime} \geq-Q_{0}$ and any $\gamma \Phi \Phi^{\prime} \geq-R_{0}$. We first show that $K C A-A=(K C-I) A$ is stable, which is equivalent to show that $A(K C-I)=A K C-A$ is stable. The equivalence is due to Lemma 3 . Since $X \in \mathbb{R}^{n \times n}$ is stable iff $-X^{\prime}$ is stable, $K C A-A$ is stable is then further equivalently to $-(A K C-A)^{\prime}$ is stable.

$$
\begin{aligned}
-(A K C-A)^{\prime} & =A^{\prime}-C^{\prime} K^{\prime} A^{\prime} \\
& =A^{\prime}-C^{\prime}\left[C P C^{\prime}+R_{0}\right]^{-1} C P A^{\prime}
\end{aligned}
$$

By part two of Lemma 4, the above matrix is stable, which proves $K C A-A$ is stable. As a consequence, Lemma 2 guarantees $\bar{P}_{\infty}(\lambda, \gamma) \geq 0$ exists and is unique for any $\lambda \Psi \Psi^{\prime} \geq-Q_{0}$ and any $\gamma \Phi \Phi^{\prime} \geq-R_{0}$.

Since $(K C A-A)$ is stable, it is clear that the second part of the theorem is true from Lemma 2.

Corollary 5: 1) If $\lambda_{1} \leq \lambda_{2}$ and $\gamma_{1} \leq \gamma_{2}$, then

$$
\bar{P}_{\infty}\left(\lambda_{1}, 0\right) \leq \bar{P}_{\infty}\left(\lambda_{2}, 0\right) \text {, and } \bar{P}_{\infty}\left(0, \gamma_{1}\right) \leq \bar{P}_{\infty}\left(0, \gamma_{2}\right)
$$

2) If $-Q_{0} \leq \lambda \Psi \Psi^{\prime} \leq 0$ and $-R_{0} \leq \gamma \Phi \Phi^{\prime} \leq 0$, then

$$
\bar{P}_{\infty}(\lambda, \gamma) \leq \bar{P}_{\infty}(0,0)
$$

3) If $\lambda \Psi \Psi^{\prime} \geq 0$ and $\gamma \Phi \Phi^{\prime} \geq 0$, then

$$
\text { Proof: 1) }
$$

$$
\bar{P}_{\infty}(\lambda, \gamma) \geq \bar{P}_{\infty}(0,0)
$$

$$
\begin{gathered}
\bar{P}_{\infty}\left(\lambda_{2}, 0\right)-\bar{P}_{\infty}\left(\lambda_{1}, 0\right)=\bar{P}_{\infty}\left(\lambda_{2}-\lambda_{1}, 0\right) \geq 0 \\
\bar{P}_{\infty}\left(0, \gamma_{2}\right)-\bar{P}_{\infty}\left(0, \gamma_{1}\right)=\bar{P}_{\infty}\left(0, \gamma_{2}-\gamma_{1}\right) \geq 0
\end{gathered}
$$

2) Notice that if $-Q_{0} \leq \lambda \Psi \Psi^{\prime} \leq 0$ and $-R_{0} \leq \gamma \Phi \Phi^{\prime} \leq 0$, then $\lambda V_{1} \leq 0$ and $\gamma V_{2} \leq 0$ where $V_{1}$ and $V_{2}$ are defined in Theorem 1. Hence we have

$$
\bar{P}_{\infty}(\lambda, \gamma)=\bar{P}_{\infty}(0,0)+\lambda V_{1}+\gamma V_{2} \leq \bar{P}_{\infty}(0,0) .
$$

3) The third part is similar to the second part.

Remark 6: Part two of Corollary 5 tells us that if the actual process and measurement noise covariances $\left(Q_{0}+\gamma \Psi \Psi^{\prime}\right.$ and $R_{0}+\lambda \Phi \Phi^{\prime}$ ) are smaller than the nominal ones used in the Kalman filter, the actual estimation error covariance will be smaller than the output from the Kalman filter. Therefore it is always better to over estimate the noise covariances than under estimate them as long as the over estimated noise covariances satisfy the required performance. This can be explained as follows. For general Kalman filter, $P_{k}$ is a function of $K_{k}$ as well as $Q_{0}, R_{0} . P_{k}$ is minimized for the $K_{k}$ given by Equ (12), assuming $Q_{0}$ and $R_{0}$ are fixed. In our analysis, $K_{k}$ is the same for different noise covariances. It is then true that $P_{k}$ will be smaller for the the one having smaller noise covariances.

\section{Sensor Fusion in a SEnsor Network}

In this section, we apply the previous results to the sensor fusion problem in a sensor network stated in Section II. We get an upper bound on the fused state estimation error. For each sensor $i=1, \cdots, N$, the associated variables (defined as in Section IV) are $\left(\hat{x}_{k}^{i}, e_{k}^{i}, P_{k}^{i}, \bar{P}_{k}^{i}, P_{\infty}^{i}, \bar{P}_{\infty}^{i}, V_{1}^{i}, V_{2}^{i}\right)$. Let the fused state estimate at time $k$ be $\hat{x}_{k}$. For ease of notation, let us define

$$
\begin{aligned}
& J \triangleq \sum_{i=1}^{N}\left(P_{k}^{i}\right)^{-1}, \\
& F_{i} \triangleq\left[I+\sum_{j=1, j \neq i}^{N} P_{k}^{i}\left(P_{k}^{j}\right)^{-1}\right]^{-1} .
\end{aligned}
$$

It can be showed that $\sum_{i=1}^{N} F_{i}=I$.

Assuming no uncertainties associated with the process or the measurement noise covariances, the optimal combined estimate can be computed using the general fusion formula [9]

$$
\hat{x}_{k}=J^{-1}\left[\sum_{i=1}^{N}\left(P_{k}^{i}\right)^{-1} \hat{x}_{k}^{i}\right]=\sum_{i=1}^{N} F_{i} \hat{x}_{k}^{i} .
$$

Now assume the uncertainties exist so that the actual error covariances $\bar{P}_{k}^{i} \neq P_{k}^{i}$. The fusion center still makes use of the fusion rule (20). We have the following result regarding the actual error covariance of the fused estimate.

Theorem 7: The actual error covariance $\bar{P}_{k}$ from the fusion rule (20) satisfies

$$
\bar{P}_{k} \leq \tilde{P}_{k} \tilde{P}_{k}^{\prime}
$$

where

$$
\tilde{P}_{k}=\sum_{i=1}^{N} F_{i}\left(\bar{P}_{k}^{i}\right)^{\frac{1}{2}}
$$

Proof: We can write the state estimation error as

$$
\begin{aligned}
e_{k} & =\left(\sum_{i=1}^{N} F_{i}\right) x_{k}-\sum_{i=1}^{N} F_{i} \hat{x}_{k}^{i} \\
& =\sum_{i=1}^{N} F_{i} e_{k}^{i} .
\end{aligned}
$$

Therefore we have

$$
\begin{aligned}
\bar{P}_{k} & =\mathrm{E}\left[e_{k} e_{k}^{\prime}\right] \\
& =\sum_{i=1}^{N} \sum_{j=1}^{N} F_{i} \mathrm{E}\left[e_{k}^{i} e_{k}^{j^{\prime}}\right] F_{j}^{\prime} \\
& \leq \sum_{i=1}^{N} \sum_{j=1}^{N} F_{i}\left(\mathrm{E}\left[e_{k}^{i} e_{k}^{i^{\prime}}\right]\right)^{\frac{1}{2}}\left(\mathrm{E}\left[e_{k}^{j} e_{k}^{j^{\prime}}\right]\right)^{\frac{1}{2}} F_{j}^{\prime} \\
& =\left[\sum_{i=1}^{N} F_{i}\left(\bar{P}_{k}^{i}\right)^{\frac{1}{2}}\right]\left[\sum_{i=1}^{N} F_{i}\left(\bar{P}_{k}^{i}\right)^{\frac{1}{2}}\right]^{\prime}
\end{aligned}
$$


where the inequality comes from Cauchy-Schwartz Inequality.

Let us define

$$
\begin{aligned}
F_{\infty}^{i} & \triangleq\left[I+\sum_{j=1, j \neq i}^{N} P_{\infty}^{i}\left(P_{\infty}^{j}\right)^{-1}\right]^{-1} . \\
\tilde{P}_{\infty}\left(\lambda, \gamma_{1}, \cdots, \gamma_{N}\right) & \triangleq \sum_{i=1}^{N} F_{\infty}^{i}\left(\bar{P}_{\infty}^{i}(0,0)+\lambda V_{1}^{i}+\gamma_{i} V_{2}^{i}\right)^{\frac{1}{2}} .
\end{aligned}
$$

Corollary 8: The actual error covariance in the steady state $\bar{P}_{\infty}$ is related to $\tilde{P}_{\infty}=\tilde{P}_{\infty}\left(\lambda, \gamma_{1}, \cdots, \gamma_{N}\right)$ as

$$
\bar{P}_{\infty} \leq \tilde{P}_{\infty} \tilde{P}_{\infty}^{\prime}
$$

Corollary 8 shows how the uncertainties in the individual sensors affect the fused estimate in the steady state. If the sensor measurements are less correlated, the upper bound is tight.

\section{EXAMPLES AND Simulations}

In this section, we provide three examples to demonstrate the theory developed so far. Example 9 considers the single sensor case and it corresponds to the results in Section IV. Example 10 considers the two sensor case and is an illustration for the results in Section V. We use the following nominal parameters for the Kalman filter in both examples.

$$
\begin{aligned}
A= & {\left[\begin{array}{cccc}
1 & 0 & 0.5 & 0 \\
0 & 1 & 0 & 0.5 \\
0 & 0 & 1 & 0 \\
0 & 0 & 0 & 1
\end{array}\right] } \\
Q_{0}= & {\left[\begin{array}{cccc}
0.0256 & 0.0039 & 0.0625 & 0.0156 \\
0.0039 & 0.0256 & 0.0156 & 0.0625 \\
0.0625 & 0.0156 & 0.2600 & 0.0625 \\
0.0156 & 0.0625 & 0.0625 & 0.2600
\end{array}\right] } \\
C= & {\left[\begin{array}{llll}
1 & 0 & 0 & 0 \\
0 & 1 & 0 & 0
\end{array}\right], R_{0}=\left[\begin{array}{ll}
1 & 0 \\
0 & 1
\end{array}\right] }
\end{aligned}
$$

The example is taken from [13] with slight modification of the process and measurement noise covariances. In order to visualize general vector cases, we plot the squared norm of the estimation errors and the trace of the computed error covariances.

Example 9: In this example, we choose $\lambda \Psi \Psi^{\prime}=0.1 I$ and $\gamma \Phi \Phi^{\prime}=2 I$, therefore the actual noise covariances are bigger than the ones used in the Kalman filter. As a result the actual error covariance $\operatorname{Tr}\left(\bar{P}_{k}\right)$ is bigger than the computed error covariance $\operatorname{Tr}\left(P_{k}\right)$ from the Kalman filter. This can be seen from Figure 2. We also plot the running average $\frac{1}{k} \sum_{i=1}^{k}\left|e_{k}\right|^{2}$ in the figure and we can see that this empirical average value converges to $\operatorname{Tr}\left(\bar{P}_{k}\right)$.

Example 10: In this example, the nominal parameters of the two sensors are

$$
\begin{aligned}
C_{1} & =C_{2}=\left[\begin{array}{llll}
1 & 0 & 1 & 0 \\
0 & 1 & 0 & 1
\end{array}\right] \\
R_{01} & =\left[\begin{array}{cc}
3 & 0 \\
0 & 0.1
\end{array}\right], R_{02}=\left[\begin{array}{cc}
0.1 & 0 \\
0 & 5
\end{array}\right]
\end{aligned}
$$

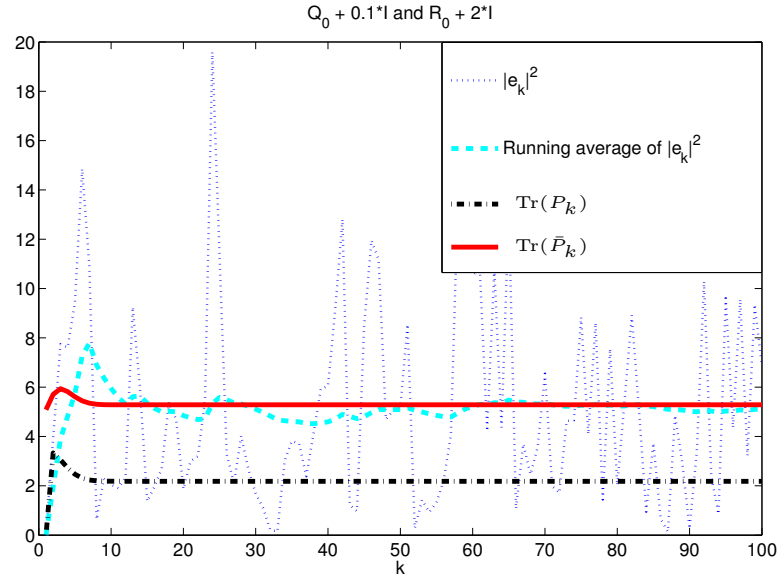

Fig. 2. Relationship between $P_{k}$ and $\bar{P}_{k}$

Notice that sensor 1 has less noise in its second output and sensor 2 has less noise in its first component. The fused estimate should therefore be better than any individual sensor, which is seen from Figure 3, where we choose $\gamma_{1} \Phi_{1} \Phi_{1}^{\prime}=\gamma_{2} \Phi_{2} \Phi_{2}^{\prime}=I$ and $\lambda \Psi \Psi^{\prime}=0.1 I$.

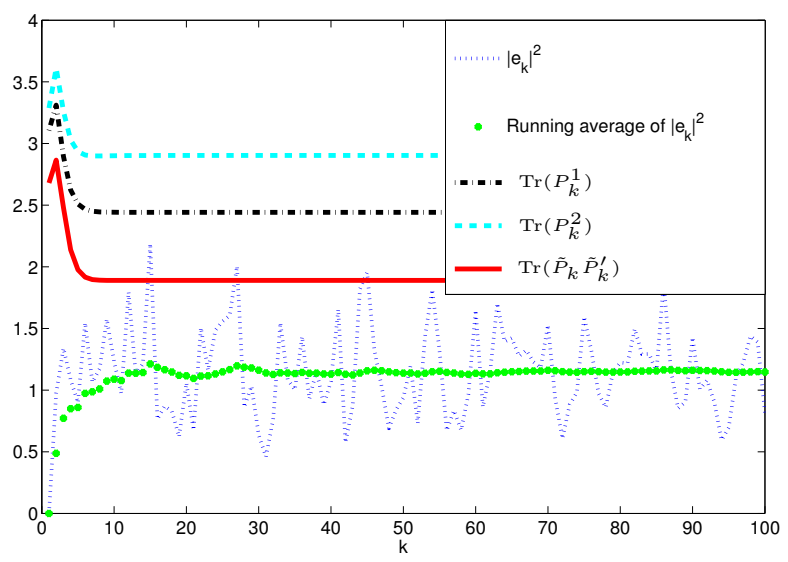

Fig. 3. Individual and fused estimates

We provide a plot below showing how the upper bound varies as the uncertainty $\gamma_{1} \Phi_{1} \Phi_{1}^{\prime}$ of $R_{1}$ increases (see Figure 4, legend for each subplot is the same as in Figure 3). It is clear that the upper bound increases as well. It is also see from the plot that, if the uncertainties in $R_{1}$ is small enough, it is guaranteed that the fused state is better than any individual ones. Once the uncertainties in sensor 1 is bigger than certain value, it is then not guaranteed that the fused state is better than using sensor 2 alone. We leave it to the future work to analytically find out this threshold value for guaranteed better fused performance due to the limited time and space here.

Example 11: A Kalman filter for estimating the road grade based on uncertain GPS readings is developed and tested in [4]. The structure of the system is similar to the setup of this paper and the uncertainty of the sensor readings, 

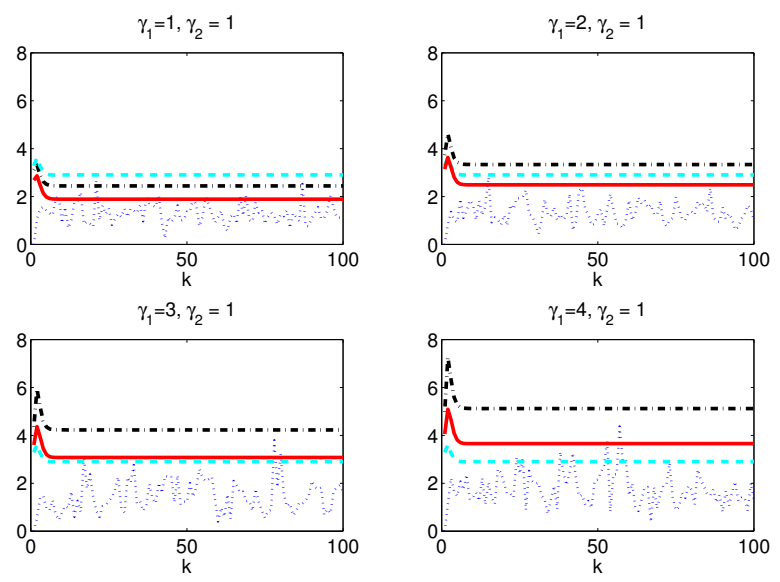

Fig. 4. Individual and fused estimates for different $\gamma_{1}$

correponding to the number of available satellites, is modelled by varying the measurement noise covariance. We test the validity of Theorem 1 by evaluating the estimation error for data collected from a segment of a Swedish highway ${ }^{1}$ for various values of the measurement noise covariance, i.e., various values of $\gamma$. Figure 5) shows the running average of the squared error over a fixed time interval as a function of the uncertainty $\gamma$. As expected from Theorem 1, there is approximately an affine relation between $\gamma$ and the variance of the estimation error.

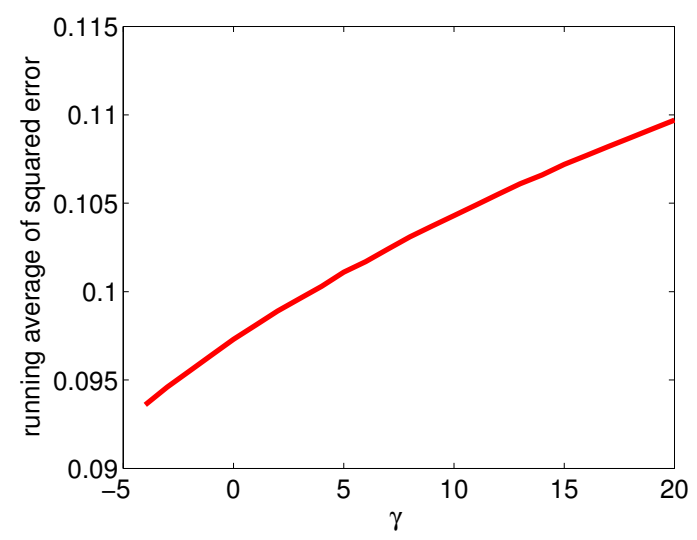

Fig. 5. Running average of the squared error as a function of the measurement noise uncertainty $\gamma$. As expected from Theorem 1, the relation is approximately affine.

\section{CONCLusion And Future Work}

In this paper, we have studied the estimation problem using Kalman filter but subject to uncertainties in the process and measurement noise covariances. Using the nominal values of the noise covariances as input to the Kalman filter, we have provided a closed form solution to how the actual error covariance evolves as a function of these uncertainties. We have also applied the theory developed in a sensor fusion problem and provided an upper bound of the estimation error covariance.

For the future work, we will study if the network that those sensors send their measurements over is bandwidth limited. In that case only a small portion of the sensors are allowed to transmit at a time. In [17], the current authors have provided several schemes for the uncertainty free cases. It will be interesting to extend that result for this work.

\section{REFERENCES}

[1] L. B. Cremean, T. B. Foote, J. H. Gillula, G. H. Hines, D. Kogan, K. L. Kriechbaum, J. C. Lamb, J. Leibs, L. Lindzey, A. D. Stewart, J. W. Burdick, and R. M. Murray, "An information-rich autonomous vehicle for high-speed desert navigation," Journal of Field Robotics, Submitted, Dec 2005.

[2] K. H. Johansson, M. Törngren, and L. Nielsen., Vehicle applications of controller area network, In D. Hristu-Varsakelis and W. S. Levine, Ed., Handbook of Networked and Embedded Control Systems, Birkäuser, 2005.

[3] N. Pettersson and K. H. Johansson, "Modeling and control of auxiliary loads in heavy vehicles," International Journal of Control, 79:5, Special issue on advanced design methodologies in automotive control, 2006.

[4] P. Sahlholm, H. Jansson, E. Kozica, and K. H. Johansson, "A sensor and data fusion algorithm for road grade estimation," in IFAC Symposium on Advances in Automotive Control, Monterey Coast, CA, USA, 2007.

[5] R.E.Kalman, "A new approach to linear filtering and prediction problems," Trasactions of ASME, Journal of Basic Engineering on Automatic Control, vol. 82(D), pp. 35-45, 1960.

[6] B.Anderson and J.Moore, Optimal Filtering. Prentice Hall, 1990.

[7] M. S. Grewal and A. P. Andrews, Kalman Filtering Theory and Practice. Prentice Hall, 1993.

[8] T.Kailath, A.Sayed, and B.Hassibi, Linear Estimation. Prentice Hall, 2000.

[9] F. Gustafsson, Adaptive Filtering and Change Detection. John Wiley \& Sons Inc, 2000.

[10] W.S.Wong and R.W.Brockett, "Systems with finite communication bandwidth-part i: State estimation problems," IEEE Trans. Automat. Contr., vol. 42, Sept 1997.

[11] B.Sinopoli, L.Schenato, M.Franceschetti, K.Poolla, M.Jordan, and S.Sastry, "Kalman filtering with intermittent observations," IEEE Transactions on Automatic Control, vol. 49, no. 9, pp. 1453-1464, 2004.

[12] X. Liu and A. Goldsmith, "Kalman filtering with partial observation losses." IEEE Control and Decision, 2004.

[13] V. Gupta, T. Chung, B. Hassibi, and R. M. Murray, "On a stochastic sensor selection algorithm with applications in sensor scheduling and dynamic sensor coverage," Automatica, vol. 42, no. 2, pp. 251-260, 2006.

[14] R. K. Mehra, "On the identification of variances and adaptive kalman filtering," IEEE Transactions on Automatic Control, vol. 15, no. 2, April 1970.

[15] R. A.Horn and C. R.Johnson, Matrix Analysis. Cambridge University Press, 1985.

[16] D. Bertsekas, Dynamic Programming and Optimal Control, vol.1. Athena Scientific, 2000.

[17] L. Shi, M. Epstein, B. Sinopoli, and R. M. Murray, "Effective sensor scheduling schemes in a sensor network by employing feedback in the communication loop." IEEE Conference on Control Applications, Singapore, To appear, 2007.

\footnotetext{
${ }^{1}$ The data were kindly provided by Per Sahlholm.
} 